\title{
2. Der psychiatrische Blick: Worauf man beim „ersten Blick" achten sollte und was man dabei erkennen kann
}

\author{
Sabina Levy und Konrad Uebelhack
}

Ein geübter diagnostischer Blick kann helfen, wichtige Entscheidungen zu treffen, wenn Eile geboten ist oder nur eine geringe Kooperationsfähigkeit der -bereitschaft seitens des Patienten besteht.

In diesen Situationen müssen Gefährdungspotenzial und Akuität eingeschätzt sowie eine Prognose erstellt werden. Sowohl Erregung und Hemmung als auch andere quantitative und qualitative Änderungen in den Kategorien Denken, Affekt und Psychomotorik müssen differenzialdiagnostisch betrachtet werden, da sie für die Diagnosestellung einen besonderen Stellenwert haben.

Die Erfahrung zeigt, dass sich der klinische Blick, die Fähigkeit zur treffenden Verbalisierung und Einordnung dessen, was Sie bei Patienten wahrnehmen, erst mit der Zeit herausbildet. Oft sind es aber gerade Anfänger, denen die Tätigkeit in Notaufnahmen und auf den Stationen obliegt, mit mehr oder weniger guter Supervision.

Viele Kollegen beschreiben zu Beginn ihrer Tätigkeit eine Überflutung mit Eindrücken, die in die bisherigen Kategorien, die sie bezüglich der Einschätzung von Menschen hatten, kaum einzuordnen sind. Dies bezieht sich sowohl auf die Intensität der Gefühle (Mitleid, Traurigkeit, Ablehnung, Ekel, Wut, Hilflosigkeit ...) als auch das gleichzeitige Bestehen von Gefühlen bzw. die Verdichtung dieser. Vor allem im Nachgang von Kontakten mit einigen Menschen mit einer Persön- 
2 Der psychiatrische Blick: Worauf man beim „ersten Blick“ achten sollte und was man dabei erkennen kann

lichkeitsstörung beschreiben viele Kollegen auch intensive unangenehme Gefühle wie Ablehnung, Ekel, Wut und empfinden es als besondere Herausforderung, nicht auf den von dem Patienten vorgegebenen Interaktionsstil einzusteigen. Gleichzeitig besteht aber der Wunsch, eben solche Gefühle den Patienten gegenüber nicht zu empfinden, um ihnen authentisch, mit Höflichkeit, Respekt und Empathie begegnen zu können.

Der Kontakt zu Patienten mit Erkrankungen aus dem schizophrenen Formenkreis weckt bei vielen Kollegen Interesse, da vieles auf den ersten Blick so anders und fremd erscheint, als würde der Patient aus einem fernen Land kommen, dessen Sprache und Kultur man schwer begreifen kann: manchmal bedrohlich, manchmal ekstatisch religiös gefärbt, manchmal bizarr und damit oft faszinierend oder irritierend.

Grundsätzlich profitiert man als Anfänger davon, möglichst viele erfahrene Kollegen bei der Exploration zu beobachten. Wie kommen erfahrene Kollegen an Informationen? Mit welchen Fragen schaffen sie es, den ersten Eindruck für andere nachvollziehbar zu verbalisieren? Wie schaffen es diese Kollegen, herausfordernde Situationen ohne starke emotionale Aufreibung zu bewältigen? In welchen Situationen fehlt es uns dennoch an dem nötigen Handwerkszeug?

Es ist wichtig, möglichst oft erfahrene Kollegen bei der Exploration zu beobachten, dabei lernt man sehr viel. Man muss nicht jeden Fehler erst selbst begehen und das Rad neu erfinden. Im Laufe der Zeit entwickelt man einen eigenen Explorations- und individuell gefärbten Stil bei der Therapie.

\section{Nachdenken über eigene Reaktionen und Reaktionen des Patienten}

Viele jüngere Kollegen berichten, dass Raum für eigene Reflexionen erst nach der ersten Zeit auf Station und des formalen Bewältigens des Arbeitspensum entsteht. Die Arbeit mit psychisch erkrankten Menschen kann sehr anstrengend und auslaugend sein. Eine gute Psychohygiene ist wichtig, um diesen Herausforderungen gewachsen zu sein. Dazu lohnt sich der Blick auf die eigenen Reaktionen und die des Patienten:

Wie reagieren wir auf Ansprüchlichkeit und herausforderndes Verhalten? Wofür sprechen diese Ansprüchlichkeit und das herausfordernde Verhalten? Was will der Patient eigentlich kommunizieren, wie kommuniziert er das? Was gibt er vor, kommunizieren $\mathrm{zu}$ wollen? Was ist, wenn trotz des Leidens jegliche „gesunde“ Ansprüchlichkeit bei ihm fehlt?

Ebenfalls wichtig ist die Frage, was in uns Sympathie und Antipathie erzeugt. Sieht man Ihnen die Antipathie an und woran? Wer erzeugt in uns Hilfsbereitschaft, und wer schafft es, uns zu instrumentalisieren? Bei wem ist unsere Hilfsbereitschaft nur professionell aufgesetzt und warum? Bei welchen Patientengruppen fühlt sich unsere Hilfsbereitschaft angemessen an? Was ist unser therapeutischer Erstimpuls bei einem Patienten? Wir reagieren hier alle unterschiedlich auf verschiedene Patienten, können mit einigen Störungsbildern besser umgehen und reagieren aversiv auf andere. 
Erfahrungsgemäß bildet sich mit der Zeit ein gutes Gefühl dafür heraus, wo eine sofortige Intervention notwendig ist, was dadurch fundierter wird, dass neben dem Erstimpuls eine gründliche Anamnese erhoben wird, die dann den ersten Eindruck stützen kann (oder ihm widerspricht) und so unsere Entscheidungen für andere nachvollziehbar macht.

Was ist die Motivation des Kommens des Patienten? (selbst- oder fremdmotiviert?)

Was ändert das an unserer Reaktionsweise und unserem Herangehen?

\section{Was verrät der „erste Blick“ (Was wir ohne explizite Exploration oder bei der Anamneseerhebung nebenbei erfassen. Was ansonsten beim ersten Kontakt zu beachten ist ...)?}

\section{Allgemeines}

Wir sind damit ausgestattet, eine Vielzahl von Informationen auf einen Blick aufnehmen zu können und intuitiv zu einem Urteil zu kommen und Entscheidungen zu treffen.

Wenn wir einen Menschen sehen, und gerade auch beim ersten Mal, achten wir auf Aussehen, Psychomotorik, Körpersprache, Hygiene, Kleidung, Stil u.v.m. Uns fallen Unstimmigkeiten zwischen äußerem Bild und dem, was sich uns im psychopathologischen Befund und der aktuellen Situation bietet, auf. Wir sehen, ob die Kleidung geschmacklich stimmig und von guter Qualität ist, ob sie gut aussieht, ob sie alt oder neu ist. Wir bekommen so manchmal mit, ob Brüche im Lebenslauf stattfanden. Auch im umgekehrten Fall der Besserung oder Genesung beobachten wir, dass sich Menschen wieder pflegen, sich frisieren, Kosmetik verwenden, Haare wieder färben, Nägel lackieren, sich wieder normal bewegen und sprechen, wieder lachen und sich für die Welt reflektiert interessieren.

Widersprüche von äußerer Erscheinung und psychopathologischem Befund können auf einen Bruch im Lebenslauf hinweisen.

Der Rückgang dieser Diskrepanzen kann Zeichen der Remission sein.

Auch die Art und die Geschwindigkeit der Sprache sollten Beachtung finden. Wir nehmen wahr, wie Aufmerksamkeit, Konzentration und deren jeweilige Spanne, sowie die Ordnung des Denkens sind, ob jemand ratlos oder verwirrt ist.

Auch bedeutet eine gute Aufmerksamkeit nicht automatisch eine gute Auffassung (eindrücklich zu beobachten bei Demenzen oder Psychosen mit ausgeprägten Denkstörungen). 
2 Der psychiatrische Blick: Worauf man beim „ersten Blick“ achten sollte und was man dabei erkennen kann

\section{Spezielle Aspekte und Hintergründe, Anregungen zur differenzierten Beobachtung für den Erstkontakt und die Anamneseerhebung}

\section{Körpersprache}

Die Körpersprache hat, ähnlich der Traumsymbolik, oft keine für alle Menschen gleich geltende Bedeutung. Vielmehr geht es um die Zusammenschau aller Signale und der Situation sowie um den soziokulturellen Hintergrund.

Bei der Einschätzung v.a. der Sprache, Körpersprache und Psychomotorik, muss eingeschätzt werden, in welchem Ausmaß hier Erlerntes, Imitiertes, Konditioniertes oder Demonstratives, insbesondere unter den Aspekten der kulturell-spirituellreligiösen oder anderweitigen ideologischen Beeinflussung, zum Tragen kommt.

Oft verrät die Körpersprache viel über die Persönlichkeit und aktuelle Probleme. Man sieht, ob mit Gestik, Mimik und Sprache, aber auch mit Kleidung, etwas demonstriert, kompensiert oder versteckt wird.

Hierbei können wir auch die Art und Stärke des Händedruckes, die Beschaffenheit und Feuchtigkeit der Haut und den Muskeltonus einschätzen. Z.B. kann ein wahnhafter Patient angeben, vollkommen entspannt zu sein, gleichzeitig kann uns aber ein erhöhter Puls und Blutdruck, dazu noch ein sehr kräftiger Händedruck auf ein dennoch vorhandenes hohes Anspannungsniveau hinweisen. Verschränkte Arme, eng übereinander geschlagene Beine können z.B. auf Verschlossenheit, Abwehr oder Frieren hinweisen.

\section{Kommunikation/Metakommunikation}

„Man kann nicht nicht kommunizieren.“ Paul Watzlawick

Folgendes sollte in der Anamnese diesbezüglich eingeschätzt werden:

- Besteht verbale/nonverbale Kommunikationsfähigkeit/-freudigkeit?

- Besteht Zugewandtheit und Offenheit?

- Prägt Oberflächlichkeit oder Tiefe die Erzählung?

- Mitteilungsbereitschaft: Ist sie unangestoßen, auf Nachfrage oder mit Zähigkeit vorhanden, besteht Verweigerung oder Unfähigkeit (z.B. aufgrund von Denkstörungen)?

- Besteht Blickkontakt? Wenn keiner gehalten wird, ist die verbal ausgesprochene Information eher zu hinterfragen als mit vorhandenem Blickkontakt. Eine möglicherweise nur eingeschränkte Verwertbarkeit der gegebenen Informationen sollte dann bedacht werden.

Die Kommunikation kann aus verschiedenen Ursachen eingeschränkt sein, hierbei sollte auch die mögliche Schwerhörigkeit im Alter bedacht werden, die oft unerkannt bleibt. 
Oft zeigt sich innerhalb der ersten Minuten eine Tendenz über die inhaltliche Verwertbarkeit der Angaben:

Berichtet der Patient geradlinig oder umständlich, schlüssig oder paralogisch? Inwieweit sind die geschilderten Inhalte einfühlbar? Besteht Mitteilungsbereitschaft? Wie ist eine mangelnde Mitteilungsbereitschaft zu werten? Entspringt sie einem Misstrauen, einer feindseligen Haltung oder der Scham?

Metakommunikation

Jede Botschaft (Sender an Empfänger) besteht aus vier Teilen (Friedemann Schulz von Thun):

- Die Sach-Aussage: Sie ist der konkrete Fakt (Worüber wird gesprochen?).

- Die Eigenoffenbarung: Sie sagt etwas über den Sender (Was wird über sich offenbart?).

- Die Beziehungsseite: Sie gibt das Verhältnis zwischen Sender und Empfänger an.

- Der Appell: Er fordert den Empfänger auf (Was will der Sender?).

Wenn der Appell benutzt wird, um die anderen drei Bereiche zu bestimmen und zu funktionalisieren, spricht man von Manipulation.

Jeder der 4 Punkte kann unterschiedlich in Erscheinung treten:

- explizit/implizit (direkt/indirekt - sowohl verbal als auch Gesten möglich)

- verbal/nonverbal

Die 4 Botschaften können dabei kongruent oder inkongruent sein.

Vereinfacht kann man sagen, dass es oft auf deutliche Diskrepanzen (Inkongruenz) zwischen den vier Teilen der Botschaft zurückzuführen ist, wenn man nach einem Gespräch Schwierigkeiten hat, dieses zu dokumentieren.

\section{Übertragung/Gegenübertragung}

Wenn sich zwei Menschen begegnen, haben sie Gefühle dem anderen gegenüber und erzeugen jeweils Gefühle im anderen.

Ein Patient kann in der Begegnung mit uns Gefühle uns gegenüber erleben, die nicht zu uns/dieser Situation mit uns passen, sondern die sich eigentlich auf eine andere Person aus seiner Vergangenheit beziehen. Dies nennt man Übertragung.

Übertragung ist eine „Wiederholung“ einer früher entstandenen Beziehung, mit Projektion von, eigentlich an jemand früheren gerichteten Gefühlen, Wünschen und Erwartungen, aber auch Befürchtungen, Triebimpulsen und Abwehrhaltungen usw. bei einer neuen Person, wie z.B. dem Arzt/Therapeuten. Diese Prozesse finden aber auch in Beziehungen des Privat- und Berufslebens (z.B. hierarchischen Beziehungen) statt und sind weitgehend unbewusst.

Es ist aber immer gleichzeitig eine Vielzahl von Gefühlen, Reaktionen, Impulsen usw. in einer solchen Situation vorhanden. Im Plural spricht man anstatt von Übertragungen auch oft von Übertragungsreaktionen. Diese sind in der aktuellen Situation immer unangemessen und verzerrt in Bezug auf die Dauer (zu lang, zu kurz) und die Ausgestaltung (zu stark, zu gering, bizarr). Aber in der vergangenen Beziehung/Erstsituation waren die damaligen Reaktionen wahrscheinlich angemessen.

Unsere Reaktion, also das Richten von Gefühlen, Wünschen, Erwartungen, Zweifel usw. an unser Gegenüber nennt man Gegenübertragung. 
2 Der psychiatrische Blick: Worauf man beim „ersten Blick“ achten sollte und was man dabei erkennen kann

Sowohl Übertragung als auch Gegenübertragung sollten von uns erkannt, gedeutet und genutzt werden können, da diese oft unbewussten Inhalte auf zum Teil verdrängte frühere soziale Beziehungen, Interaktionen und in diesen Beziehungen wurzelnde Konflikte hinweisen und unbewusst auf neue soziale Beziehungen übertragen werden. So können im therapeutischen Kontext auch Rollenerwartungen auf uns übertragen werden (z.B. an eine Vater- oder eine Mutterfigur).

Durch Selbsterfahrung lernen wir, zu unterscheiden, welche Gefühle und Reaktionsweisen wir selbst in eine Situation mit hineinbringen und welche Gefühle und Reaktionen erst bei einer Interaktion in uns erzeugt/reaktiviert werden.

Oft ist gerade der in Ausbildung befindliche Arzt in eine Hierarchie eingebettet und befindet sich nicht nur den Patienten gegenüber in einer solchen Übertragungs-/Gegenübertragungssituation, sondern auch gegenüber seinen Vorgesetzten, aber auch anderen Berufsgruppen gegenüber, wie der Pflege, deren Mitarbeitern er weisungsberechtigt ist. Hier ergeben sich auch Konstellationen gegenseitiger Erwartungen, die auch auf den Patientenkontakt Einfluss haben können.

\section{Abwehrmechanismen}

Nicht alle unserer Konflikte, Wünsche, Bedürfnisse, Werte und Muster sind uns bewusst. Viele sind unbewusst.

Intrapsychische Abwehrmechanismen sollen uns vor dem Bewusstwerden von unangenehmen und bedrohlichen Gefühlen und Affekten, die z.B. im Zusammenhang mit einem Konflikt oder Erlebnis stehen, schützen, und sie deshalb im Unbewussten halten. Die einzelnen Abwehrmechanismen entstehen in unterschiedlichen Entwicklungsphasen des heranwachsenden Menschen. Am Reifegrad des jeweiligen unbewusst zum Einsatz gekommenen Abwehrmechanismus können wir auf das Problemalter (in dem z.B. der Konflikt entstand, ein unangenehmes prägendes Ereignis stattfand) und die Reife von Persönlichkeitsanteilen und das sozioemotionale Entwicklungsniveau dieser Anteile schließen.

Eine fast zu sehr vereinfachte Grundregel für den Einstieg: Je mehr ein Abwehrmechanismus bei einem Menschen $\mathrm{zu}$ einer desintegrierten, polarisierten oder verzerrten Wahrnehmung/Beurteilung von Situationen/Personen führt, desto unreifer ist dieser Abwehrmechanismus und deutet auf eine umso frühere Entstehung der Störung (frühe Störung) hin. Hier ist aber die tiefgehende Kenntnis der Abwehrmechanismen für den klinischen/therapeutischen (und auch privaten) Alltag von unschätzbarem Wert.

Grundsätzlich können bei vielen psychischen Störungen in der akuten Krankheitsphase vorübergehend einzelne Persönlichkeitszüge oder Reaktionsweisen stärker akzentuiert sein.

Grundsätzlich können unterschiedliche Anteile eines Menschen sehr unterschiedliche sozioemotionale Entwicklungsniveaus aufweisen! 
Für die Diagnostik und Therapie bei einem Patienten und den Umgang mit ihm, sind diese Kenntnis und deren Anwendung sehr erhellend und hilfreich.

\section{Primärer und sekundärer Krankheitsgewinn bei der Symptombildung (z.B. bei neu- rotischen Störungen, Konversion, dissoziativen Störungen, Phobien)}

Bei der Beurteilung von Symptomen ist immer auch neben der möglichen organischen Genese eine psychodynamische bedingte Entstehung oder eine Kombination beider zu erwägen.

Symptome können auch unbewusst gebildet werden, um unbewusste Konflikte abzuwehren.

Die Symptombildung ist der primäre Krankheitsgewinn, da das Symptom, wenn auch unangenehm, so doch besser ertragbar ist als der abgewehrte Konflikt. Durch das Symptom kann es vermieden werden, unangenehmen Situationen und Auseinandersetzungen zu begegnen oder Entscheidungen treffen zu müssen.

Oft weist das Symptom symbolisch auf das Grundproblem des Konfliktes hin (z.B. histrionische Blindheit: etwas nicht sehen wollen oder Lähmung: zu/von etwas/jemanden nicht gehen oder weggehen wollen).

Der sekundäre Krankheitsgewinn erwächst aus der angenehmen (z.B. aufmerksamen oder fürsorglichen) Reaktion der Umgebung (unbewusst erwünscht oder bewusst erwünscht).

Zwei Fragen stellen sich hierbei:

- Was ermöglicht das Symptom dem Patienten? (Welchen Gewinn hat er dadurch primär und sekundär?)

- Was braucht der Patient wegen des Symptoms nicht mehr zu machen? (Was kann er vermeiden?)

Es gibt sogenannte Grundkonflikte, die nicht aus einer aktuellen Situation erwachsen, sondern, oft aus frühen Entwicklungsphasen stammend, einen überdauernden dysfunktionalen Charakter haben:

- Individuation versus Abhängigkeit, Unterwerfung versus Kontrolle, Versorgung versus Autarkie

- Selbstwertkonflikt, Schuldkonflikt, ödipaler Konflikt, Identitätskonflikt

\section{Proxemik}

Proxemik ist die Lehre vom Signalaustausch von Individuen durch Einnahme bestimmter Distanzen zueinander.

Die Abstände sind kultur-/subkulturabhängig und zustandsabhängig. In „unserer Kultur“ in Deutschland gelten in etwa folgende Distanzen:

- Intimdistanz: unter ca. $45 \mathrm{~cm}$

- persönliche Distanz: ca. 45 bis $120 \mathrm{~cm}$

- soziale Distanz: ca. 120 bis $360 \mathrm{~cm}$ 
2 Der psychiatrische Blick: Worauf man beim „ersten Blick“ achten sollte und was man dabei erkennen kann

- öffentliche Distanz: ab ca. 360 cm

Es gibt kulturell, individuell und situativ bedingte Wohlfühldistanzen.

Die Distanzen sind auch aktiv und passiv unterschiedlich:

- Welche Distanz wählt jemand selbst zum Gegenüber und

- welche Distanz lässt er beim Eindringen eines anderen in seinen Bereich zu?

Hier kann es deutliche Unterschiede der aktiven und passiven Distanzen geben, insbesondere bei erregten Menschen.

Wichtig ist also, den Wohlfühlbereich/die Wohlfühldistanz eines Menschen zu erkennen und nach Möglichkeit einzuhalten!

Erregte bzw. leicht erregbare, gewalttätige oder ängstliche Personen haben oft einen größeren Raum nötig, sehr misstrauische und/oder wahnhafte Patienten fühlen sich oft ebenso mit einer größeren Entfernung sicherer. Ein „Eindringen“ in diesen Raum, z.B. bei der körperlichen Untersuchung, sollte insbesondere bei misstrauischen, unruhigen Patienten immer angekündigt und erklärt werden, bei hochgradig erregten Patienten muss unter Umständen darauf verzichtet werden bzw. auf basale Untersuchungen begrenzt werden.

\section{Die Einschätzung der Suizidalität}

\section{Suizidalität sollte immer direkt besprochen werden.}

Die Suizidalität wird durch die Thematisierung nicht verstärkt.

Wenn wir das Gefühl haben, dass wir nach einem Gespräch eine Last tragen, dass der Patient sich aber entlasten konnte, ist dies eher ein gutes Zeichen. Wenn wir dagegen ein Gefühl von Leere haben und wir die Abgabe von Belastung an uns nicht spüren können, dann ist das eher ein Alarmsignal. Manchmal spüren wir echte Besorgnis, sind empathisch und werden deshalb schnell aktiv, den Patienten zu versorgen und manchmal spüren wir Desinteresse und Gleichgültigkeit in uns.

Wenn die Dramatik der Schilderung und Heftigkeit der angegebenen Suizidalität im krassen Gegensatz zu der bei uns ausgelösten Gefühle (s.o.: wie z.B. Gleichgültigkeit, keine Besorgnis) steht, sollte man nach Möglichkeit eine Zweitmeinung (am besten Facharzt) einholen und auch eine Instrumentalisierung der Suizidalität nicht ausschließen.

Hier ist insbesondere auch die Prospektivität, also die realistischen Pläne und Ideen für eine Zukunft, abzuklären, da dies ein guter Marker für die Distanzierung von Suizidalität ist. Bei der Einschätzung der Suizidalität und Prospektivität ist auch zu fragen, ob Kinder, Haustiere oder andere Personen zu versorgen sind. Auch ist zu prüfen, ob eine Fremdgefährdung (z.B. Pläne für einen erweiterten Suizid, oder Inkaufnahme von „Kollateralschäden“) vorliegt. 
IV Der klinische Blick - klinische Basisfertigkeiten und darüber hinaus

\section{Literaturempfehlung}

Reischies FM (2007) Merkmale psychischer Krankheitsbilder und klinische Neurowissenschaft. Springer Heidelberg 